\title{
Intakes and sources of dietary sugars in a representative sample of Irish adults (18-90y)
}

\author{
J. Walton ${ }^{1}$, L Kehoe ${ }^{1}$, B.A. McNulty ${ }^{2}$, A.P. Nugent ${ }^{2}$ and A. Flynn ${ }^{1}$ \\ ${ }^{1}$ School of Food and Nutritional Sciences, University College Cork, Republic of Ireland and \\ ${ }^{2}$ UCD Institute of Food and Health, University College Dublin, Belfield, Dublin 4, Republic of Ireland
}

Following recent reviews of the evidence relating carbohydrate intake to health, updated dietary guidance has become available on intake of sugars ${ }^{(1,2)}$. The World Health Organisation (WHO) recommend that intake of free sugars should be $<10 \%$ of total energy $(\% \mathrm{TE})$ and have a conditional recommendation of $<5 \% \mathrm{TE}{ }^{(1)}$. The Scientific Advisory Committee on Nutriton (SACN) (UK) also recommend that the population intake of free sugars should not exceed $5 \%$ TE for those aged 2 years and over ${ }^{(2)}$. 'Free' sugars include all mono- and di-saccharides added to foods by the manufacturer, cook or consumer, plus sugars naturally present in honey, syrups and unsweetened fruit juices ${ }^{(2)}$. There are currently few data available on intakes of free sugars in adults across Europe. The objective of this study was to estimate the intake and sources of dietary sugars (including free sugars) in Irish adults using data from the Irish National Adult Nutrition Survey (NANS) 2008-2010 (www.iuna.net) which used a 4d semi-weighed food diary to collect detailed food and beverage intake data in a representative sample of 1500 adults. Energy and total sugar values were derived from the UK Food Composition Tables ${ }^{(3)}$ and product labels. Added and free sugars values were assigned to each food and beverage consumed by adapting a systematic approach used to calculate added sugar content in foods and beverages ${ }^{(4)}$. Mean intakes of total, added and free sugars were estimated via the NCI-method ${ }^{(5)}$ using SAS ${ }^{\mathcal{C}}$ Enterprise Guide and the key sources of free sugars were calculated using SPSS ${ }^{\mathcal{C}}$ software. The table below presents the mean intakes of total, added and free sugars (g, \% TE) for the total population and split by gender and age-group.

\begin{tabular}{|c|c|c|c|c|c|c|c|c|c|c|c|c|c|}
\hline & \multirow[b]{3}{*}{$n$} & \multicolumn{4}{|c|}{ Total sugars } & \multicolumn{4}{|c|}{ Added sugars } & \multicolumn{4}{|c|}{ Free Sugars } \\
\hline & & \multicolumn{2}{|c|}{$\mathrm{g}$} & \multicolumn{2}{|c|}{$\% \mathrm{TE}$} & \multicolumn{2}{|c|}{$\mathrm{g}$} & \multicolumn{2}{|c|}{$\% \mathrm{TE}$} & \multicolumn{2}{|c|}{$\mathrm{g}$} & \multicolumn{2}{|c|}{$\% \mathrm{TE}$} \\
\hline & & Mean & $\overline{\mathrm{SD}}$ & Mean & $\overline{\mathrm{SD}}$ & Mean & $\overline{\mathrm{SD}}$ & Mean & $\overline{\mathrm{SD}}$ & $\overline{\text { Mean }}$ & $\overline{\mathrm{SD}}$ & Mean & $\overline{\mathrm{SD}}$ \\
\hline \multicolumn{14}{|l|}{ All } \\
\hline $18-90 y$ & 1500 & $90 \cdot 6$ & $38 \cdot 7$ & $17 \cdot 1$ & $5 \cdot 1$ & $43 \cdot 3$ & $28 \cdot 3$ & $7 \cdot 9$ & $4 \cdot 3$ & 47.9 & $30 \cdot 2$ & 8.7 & $4 \cdot 6$ \\
\hline $18-35 y$ & 531 & $95 \cdot 1$ & $40 \cdot 3$ & $16 \cdot 8$ & $5 \cdot 0$ & 49.9 & $30 \cdot 5$ & 8.7 & $4 \cdot 6$ & $55 \cdot 7$ & 32.7 & 9.7 & 4.8 \\
\hline $36-50 y$ & 437 & $87 \cdot 4$ & $37 \cdot 8$ & $16 \cdot 5$ & 4.9 & $41 \cdot 1$ & $27 \cdot 0$ & 7.5 & $4 \cdot 1$ & $45 \cdot 1$ & $28 \cdot 5$ & 8.2 & $4 \cdot 3$ \\
\hline $51-64 y$ & 306 & $90 \cdot 5$ & $38 \cdot 0$ & $17 \cdot 4$ & $5 \cdot 2$ & $39 \cdot 2$ & $26 \cdot 1$ & $7 \cdot 2$ & $4 \cdot 1$ & $43 \cdot 2$ & $27 \cdot 7$ & 8.0 & $4 \cdot 3$ \\
\hline$>65 y$ & 226 & $86 \cdot 3$ & $36 \cdot 5$ & $18 \cdot 3$ & $5 \cdot 3$ & $37 \cdot 3$ & $24 \cdot 9$ & $7 \cdot 5$ & $4 \cdot 2$ & 41.2 & $26 \cdot 5$ & $8 \cdot 3$ & 4.4 \\
\hline \multicolumn{14}{|l|}{ Men } \\
\hline $18-90 y$ & 740 & $99 \cdot 1$ & $40 \cdot 1$ & $16 \cdot 1$ & $4 \cdot 9$ & $47 \cdot 5$ & 29.8 & $7 \cdot 6$ & $4 \cdot 2$ & $52 \cdot 7$ & 31.9 & 8.4 & $4 \cdot 4$ \\
\hline $18-35 y$ & 276 & 105.6 & 41.6 & $16 \cdot 0$ & 4.9 & 53.9 & $32 \cdot 0$ & $8 \cdot 1$ & $4 \cdot 3$ & $60 \cdot 7$ & $34 \cdot 3$ & $9 \cdot 2$ & 4.7 \\
\hline $36-50 y$ & 205 & $97 \cdot 1$ & $39 \cdot 1$ & $15 \cdot 8$ & $4 \cdot 8$ & $46 \cdot 4$ & 28.8 & 7.5 & $4 \cdot 1$ & $50 \cdot 3$ & $30 \cdot 2$ & 8.2 & $4 \cdot 3$ \\
\hline $51-64 y$ & 153 & $96 \cdot 5$ & $39 \cdot 1$ & $16 \cdot 2$ & 4.9 & $43 \cdot 1$ & $27 \cdot 5$ & $7 \cdot 1$ & $4 \cdot 0$ & $47 \cdot 6$ & $29 \cdot 1$ & 7.9 & 4.2 \\
\hline$>65 y$ & 106 & 89.5 & $37 \cdot 0$ & $16 \cdot 8$ & 4.9 & $39 \cdot 5$ & 25.7 & $7 \cdot 2$ & $4 \cdot 0$ & $43 \cdot 1$ & $27 \cdot 1$ & 7.8 & 4.2 \\
\hline \multicolumn{14}{|l|}{ Women } \\
\hline $18-90 y$ & 760 & $82 \cdot 3$ & $35 \cdot 4$ & $18 \cdot 0$ & $5 \cdot 2$ & $39 \cdot 1$ & $26 \cdot 0$ & $8 \cdot 1$ & $4 \cdot 4$ & $43 \cdot 2$ & $27 \cdot 7$ & 9.0 & 4.6 \\
\hline $18-35 y$ & 255 & 83.8 & $35 \cdot 5$ & $17 \cdot 8$ & $5 \cdot 1$ & $45 \cdot 6$ & $28 \cdot 2$ & $9 \cdot 2$ & $4 \cdot 7$ & $50 \cdot 3$ & 29.9 & $10 \cdot 2$ & 4.9 \\
\hline $36-50 y$ & 232 & $78 \cdot 8$ & $34 \cdot 4$ & $17 \cdot 1$ & $5 \cdot 0$ & $36 \cdot 4$ & $24 \cdot 4$ & $7 \cdot 5$ & $4 \cdot 1$ & $40 \cdot 2$ & $26 \cdot 0$ & $8 \cdot 3$ & 4.3 \\
\hline $51-64 y$ & 153 & $84 \cdot 4$ & $35 \cdot 8$ & $18 \cdot 7$ & $5 \cdot 2$ & $45 \cdot 4$ & $24 \cdot 0$ & $7 \cdot 4$ & $4 \cdot 1$ & 38.8 & 25.4 & $8 \cdot 1$ & $4 \cdot 3$ \\
\hline$>65 y$ & 120 & 83.4 & $35 \cdot 8$ & $19 \cdot 6$ & 5.4 & $35 \cdot 3$ & $24 \cdot 1$ & $7 \cdot 7$ & $4 \cdot 3$ & 39.5 & $25 \cdot 8$ & $8 \cdot 8$ & 4.5 \\
\hline
\end{tabular}

In the total population, mean intakes of total, added and free sugars are $17 \cdot 1,7.9$ and $8.7 \% \mathrm{TE}$, respectively. The mean intake of free sugars $(9 \% \mathrm{TE})$ is in line with the WHO guideline of $<10 \% \mathrm{TE}$ but exceeds the UK and (conditional) WHO recommendation of $<5 \%$ TE. The key food groups contributing to intake of free sugars are 'sugars, syrups and jams' (18\%), 'biscuits, cakes \& pastries' $(15 \%)$, sugar-sweetened beverages $(14 \%)$, fruit juice $(8 \%)$, chocolate $(8 \%)$, yogurt $(7 \%)$, breakfast cereals $(7 \%)$ and 'chilled desserts \& ice-cream' $(7 \%)$. These findings provide valuable data on the intake and sources of dietary sugars in Irish adults.

This research was funded by the Department of Agriculture, Food and the Marine under the Nutridata Project 13F 542; $2014-2016$.

1. WHO (2015) WHO. Geneva: Switzerland.

2. PHE (2015) SACN. London: TSO.

3. FSA (2002) Cambridge: Royal Society of Chemistry.

4. Louie JCY, Moshtaghian, Boylan S et al. (2015) EJCN 69, 154-161.

5. Tooze JA, Kipnis V, Buckman DW et al. (2010) Stat Med 29, 2857-2868. 